
\title{
25 Research Square \\ Independent Effects of Kidney Function and Cholesterol Efflux on Cardiovascular Mortality
}

Andreas Ritsch ( $\square$ andreas.ritsch@i-med.ac.at)

Medical University of Innsbruck

Monika Hunjadi

Medical University of Innsbruck

Tatjana Stojakovic

Medical University of Graz

Stephen Zewinger

Saarland University Medical Center

Thimoteus Speer

Saarland University Medical Center

Jürgen $E$. Scherberich

Klinikum Muenchen-Harlaching, Teaching Hospital of the Ludwig-Maximilians University

Guenther Silbernagel

Medical University of Graz

Hubert Scharnagl

Medical University of Graz

Marcus E. Kleber

Heidelberg University

Winfried März

Medical University of Graz

\section{Research Article}

Keywords: cardiovascular risk, cholesterol efflux capacity, eGFR, high density lipoprotein, kidney function, uromodulin.

Posted Date: September 2nd, 2021

DOl: https://doi.org/10.21203/rs.3.rs-828027/v1

License: (9) (1) This work is licensed under a Creative Commons Attribution 4.0 International License. Read Full License 


\section{Abstract}

\section{Background}

Impaired renal function is associated with cardiovascular and all-cause mortality. In the general population, HDL-cholesterol is associated with cardiovascular events, which is not true in patients with chronic kidney disease (CKD). This has been attributed to abnormal HDL function in CKD.

\section{Methods}

In this study, we analyzed the association between kidney function, cholesterol efflux capacity as one of the major HDL functions and cardiovascular mortality in 2469 patients of the Ludwigshafen Risk and Cardiovascular Health Study who all underwent coronary angiography.

\section{Results}

We found a strong association between cholesterol efflux capacity and kidney function. Additionally, a genetic score of 53 SNPs associated with GRF and the uromodulin SNP rs12917707 was correlated inversely with cholesterol efflux. However, adjustment for eGFR and uromodulin as markers of kidney function did not affect the relationship between cholesterol efflux and cardiovascular mortality.

\section{Conclusions}

We suggest that impaired renal function lowers cholesterol efflux, but that this is not mediating the effects of impaired kidney function on cardiovascular mortality. Other mechanisms of low cellular cholesterol efflux may causally be involved in adverse cardiovascular outcomes.

\section{Trial registration}

The study was approved by the Ethics Committee at the "Aerztekammer Rheinland-Pfalz" and was performed conform to the declaration of Helsinki (837.255.97 [1394], approved January $\left.8^{\text {th }}, 1999\right)$.

\section{Background}

Patients with chronic kidney disease (CKD) disclose a high burden for cardiovascular disease (CVD). Even mild kidney dysfunction increases the risk of cardiovascular disease.[1] In addition, a reduction in glomerular filtration rate has been identified as potent and independent risk factor.[2] Low levels of high density lipoprotein cholesterol (HDL-C) indicate a risk for atherosclerotic cardiovascular disease in the general population.[3] However, there is a growing body of evidence that raising HDL-C levels may not be atheroprotective throughout. Patients with genetically elevated apoA-I and HDL-C do not have a reduced risk for cardiovascular disease.[4] Intervention studies of CETP inhibitors or niacin (in combination with statins) had been stopped because they failed to show a beneficial effect on primary endpoints.[5] In addition, higher HDL-C levels did not associate with reduced mortality risk and CVD in patients with 
reduced kidney function.[6] These observations do not support a causal role of HDL-C in atherosclerosis and may suggest the presence of other actions of HDL particles not readily reflected by their cholesterol content. A key function of HDL among numerous actions proposed in the last years is the ability to promote the efflux of cholesterol from peripheral cells and to initiate shuttling of cholesterol back to the liver. The reverse cholesterol transport is considered atheroprotective by transferring excess cholesterol from peripheral cells back to the liver where it is secreted into the bile or converted into bile acids.

Cholesterol efflux from cholesterol laden macrophages represents an initial step within this pathway and has been shown to prevent atherosclerosis in animal models. [7] HDL dysfunction including reduced cholesterol efflux capacity has been shown to begin in early CKD and to progress with declining renal function. $[8,9]$ However, studies regarding cholesterol efflux capacity in CKD patients yielded discrepant results.[10-13] We recently reported on the association between cholesterol efflux capacity and cardiovascular risk in patients undergoing coronary angiography.[14] As these patients represent a particular population of patients with a high prevalence of CKD we investigated the influence of kidney function on cholesterol efflux capacity and cardiovascular mortality in this clinical scenario.

\section{Methods}

\section{Study Design and Participants}

We studied 2468 participants of the LUdwigshafen RIsk and Cardiovascular Health (LURIC) study.[15] 1032 patients ( $29 \%$ ) of the original study population were not included in this study as not enough sample volume of serum required for cholesterol efflux measurement had been available from these patients. Inclusion criteria were: German ancestry, clinical stability except for acute coronary syndromes, and the availability of a coronary angiogram. The indications for angiography in individuals in clinically stable condition were chest pain and/or noninvasive test results consistent with myocardial ischemia. Individuals suffering from acute illness other than acute coronary syndromes, chronic non-cardiac diseases, or malignancy within the past 5 years and subjects unable to understand the purpose of the study were excluded. The study was approved by the Ethics Committee at the "Aerztekammer RheinlandPfalz" and was performed conform to the declaration of Helsinki (837.255.97 [1394], approved January 8th, 1999). Informed written consent was obtained from all participants. Coronary artery disease (CAD) was assessed by angiography with maximum luminal narrowing estimated by visual analysis. Clinically relevant CAD was defined as the occurrence of $\geq 1$ stenosis of $\geq 20 \%$ in $\geq 1$ of 15 coronary segments. Individuals with stenoses $<20 \%$ were considered as not having CAD. Diabetes mellitus was diagnosed when plasma glucose was $>1.25 \mathrm{~g} / \mathrm{L}$ in the fasting state or $>2.00 \mathrm{~g} / \mathrm{L} 2 \mathrm{~h}$ after an oral glucose load,[16] or when antidiabetic medical treatment was prescribed. Hypertension was diagnosed when the systolic and/or diastolic blood pressure exceeded 140 and/or $90 \mathrm{~mm} \mathrm{Hg}$, respectively, or when a patient was on antihypertensive medication. Information on vital status was obtained from local registries. Among the 2468 persons studied, 717 deaths $(29.0 \%)$ occurred during a median follow-up of 9.9 (8.5-10.7) years. Cardiovascular death included sudden death, fatal myocardial infarction, death due to congestive heart 
failure, death immediately after intervention to treat CAD, fatal stroke, and other causes of death due to CAD.

\section{Laboratory Procedures}

Fasting blood samples were collected prior to angiography. The standard laboratory methods have been described. Estimated glomerular filtration rate (eGFR) was calculated using the 2012 CKD-EPI eGFR $_{\text {creat-cys }}$ equation as previously described.[6, 17] eGFR could not be calculated for 5 patients due to missing data for completion of the respective formula. Serum uromodulin was measured using a sensitive ELISA specifically adapted to serum specimens.[18]

\section{Cholesterol efflux capacity}

Cholesterol efflux capacity was quantified in blood samples as described.[19] Briefly, J774 cells, derived from a murine macrophage cell line, were plated and radiolabeled with $2 \mu \mathrm{Ci}$ of $3 \mathrm{H}$-cholesterol per milliliter. Cells were incubated with 0.3 mM cAMP (C3912, Sigma-Aldrich) to upregulate ABCA1. Subsequently, efflux medium containing 2.8\% apolipoprotein B-depleted serum was added for 4 hours. All steps were performed in the presence of $2 \mu \mathrm{g}$ per milliliter acyl-coenzyme A:cholesterol acyltransferase inhibitor (Sc-215839A, Santa-Cruz Biotechnology). Liquid scintillation counting was used to quantify the efflux of radioactive cholesterol from the cells. Percent efflux was calculated by the following formula: [(microcuries of $3 \mathrm{H}$-cholesterol in medium containing $2.8 \%$ apolipoprotein $\mathrm{B}$-depleted serum - microcuries of $3 \mathrm{H}$-cholesterol in serum-free medium) / microcuries of $3 \mathrm{H}$-cholesterol in cells extracted before the efflux step] $\times 100$. To correct for inter-assay variation across plates, a pooled serum control was included on each plate. Values for serum samples from patients are given in percentage of this control (\% C). All assays were performed in triplicate.

\section{Statistical Analysis}

Normally distributed data are presented as mean \pm SD. CETP, triglycerides, adiponectin, interleukin-6 (IL-6), and C-reactive protein (CRP) exhibited a skewed distribution and are presented as median and interquartile (Q1, Q3) range. Parameters not normally distributed were transformed logarithmically for statistical analyses. The $\chi^{2}$ test and Analysis of Variance were used to compare the distributions of the variables across the eGFR categories. The effects of cardiovascular risk factors, CAD-status and intake of lipid-lowering drugs on cholesterol efflux levels were determined using general linear models using cholesterol efflux as the dependent variable and age, lipid lowering therapy, CAD-status, body mass index (BMI), diabetes mellitus, smoking history (never, former, current), LDL-C/HDL-C ratio, and triglycerides as independent variables. Multivariate adjustments were performed for age, gender, intake of statins, CADstatus [none, stable CAD, unstable CAD, non-ST-elevation myocardial infarction (NSTEMI) or ST-elevation myocardial infarction (STEMI)], BMI, smoking status, LDL-C/HDL-C ratio, triglycerides, and metabolic syndrome / type 2 diabetes mellitus. A genetic score was calculated based on 53 independent SNPs related to eGFR. The effects of eGFR and cholesterol efflux capacity on the SNP-score corresponding to the sum of eGFR increasing alleles were determined using general linear models using the SNP-score as the dependent variable and age, gender, lipid lowering therapy, CAD-status, body mass index (BMI), 
diabetes mellitus, smoking history (never, former, current), hypertension, LDL-C/HDL-C ratio, apolipoprotein Al and triglycerides as independent variables. Cox proportional hazard models were used to examine the effect of cholesterol efflux capacity on mortality. Multivariable adjustment was performed for intake of lipid lowering drugs, age, gender, CAD, BMI, diabetes mellitus, smoking, LDL-C/HDL-C ratio and triglycerides. Moreover, the interaction term between cholesterol efflux and eGFR as well as uromodulin with regard to cardiovascular mortality was studied including the respective interaction terms as covariates. Additionally, residuals from linear regression models of eGFR as well as uromodulin on cholesterol efflux capacity were used instead of efflux capacity in the Cox proportional hazard models to test for a possible interplay between parameters of kidney function and cholesterol efflux capacity. All statistical tests were 2-sided; $P<0.05$ was considered significant. The SPSS 22.0 statistical package (SPSS Inc.) was used.

\section{Results}

\section{Study participants}

Serum samples from 2468 individuals from the LURIC study were available for measurement of cholesterol efflux capacity. Clinical and biochemical characteristics of the study population classified into three categories by their estimated GFR are presented in Table 1. Patients with moderately to severely impaired kidney function (eGFR $<60 \mathrm{ml} / \mathrm{min}$ per $1.73 \mathrm{~m}^{2}$ ) displayed typical features of uremic dyslipidemia including lower LDL cholesterol (LDL-C) and HDL-C, and higher triglycerides. This was accompanied by decreased concentrations of ApoA-I and ApoA-II, but unchanged ApoB. These data are consistent with the increased levels of CETP in patients with low eGFR. No differences in use of lipid lowering drugs were observed, but the rates of diabetes mellitus and of female sex were higher in patients with severe kidney failure (Table 1). During a median follow up time of 9.9 years, 717 participants (29.2 $\%)$ died, $62.5 \%(n=448)$ of all deaths were due to cardiovascular causes.

\section{Cholesterol efflux capacity and kidney function}

We found a strong association between cholesterol efflux capacity and eGFR in our study population. Notably, even in patients with moderately reduced renal function, cholesterol efflux capacity was reduced as compared to those with normal renal function (Fig. 1, left panel). These results were confirmed using serum uromodulin, which has recently been shown to be a useful marker for cardiovascular and renal health (Fig. 1, right panel).[20] In subgroup analyses we showed that the association between eGFR and cholesterol efflux was stronger in men, whereas the association between serum uromodulin and efflux was much stronger in women. Both associations did not depend on HDL-C plasma concentrations.

In a recent genome-wide association study combining data from 133,413 individuals with replication in 42,166 individuals 53 loci associated with eGFR were found.[21] Identified genes were enriched for expression in kidney tissues and in pathways relevant to kidney development, structure and function. In the current study, a genetic score based on all 53 SNPs was higher in control patients when compared to patients with moderate kidney insufficiency and lower in patients of the lowest cholesterol efflux quartile (Fig. 2, upper panel). This is supported by parallel investigations of the association of the uromodulin 
related polymorphism rs12917707 with serum uromodulin and the cholesterol efflux capacity, respectively (Fig. 2, lower panel).

\section{Cholesterol efflux capacity, kidney function and mortality}

Among the 2468 persons studied, 717 deaths (29.2\%) occurred during a median follow-up of 9.9 years. No associations were found between cholesterol efflux capacity and total mortality in adjusted models (Table 2, all-cause mortality, models 2 and 3). However, in patients within the lowest quartile of cholesterol efflux, the multivariably adjusted risk for cardiovascular death was significantly increased (Table 2, cardiovascular mortality, model 2). The prognostic value of cholesterol efflux capacity was also seen after further adjustment for the intake of lipid-lowering drugs, cardiovascular risk factors, CAD status and diabetes mellitus (Table 2, cardiovascular mortality, model 3).

Interestingly, inclusion of eGFR or serum uromodulin into the respective models did not reveal a major impact on the association between cholesterol efflux capacity and cardiovascular mortality (Table 2, Cardiovascular mortality, models 4 and 7). The same was observed when we included genetic variants related to either eGFR or uromodulin (Table 2, cardiovascular mortality, models 5 and 8 ). We also assessed a possible interaction between cholesterol efflux and kidney function parameters. Inclusion of interaction terms of cholesterol efflux capacity on the one hand and eGFR or serum uromodulin on the other hand did not alter the prognostic value of cholesterol efflux capacity (Table 2, cardiovascular mortality, models 6 and 9).

Finally, we analyzed residuals obtained from linear regression analysis of cholesterol efflux (dependent variable) and eGFR or serum uromodulin (independent variables), respectively. These residuals showed similar associations with cardiovascular mortality as seen in previous investigations using unadjusted cholesterol efflux capacity (Table 3 ).

\section{Discussion}

In this study, we analyzed cholesterol efflux capacity as one of the major HDL functions in patients who underwent coronary angiography. Patients were followed for a median of 9.9 years. We found a strong association between cholesterol efflux capacity and kidney function in our study population. Cholesterol efflux capacity was associated with cardiovascular mortality. A genetic score based on 53 SNPs related to eGFR, as well as the uromodulin related polymorphism rs 12917707 were shown to be associated with cholesterol efflux capacity, but these genetic variants did not relate to CVD mortality. Because, in addition, cholesterol efflux capacity adjusted for eGFR or uromodulin was invariably associated with CVD mortality, we conclude that cholesterol efflux is not a mediator of adverse cardiovascular outcomes in renal insufficiency.

Patients with chronic kidney disease are at dramatically increased risk for CAD, which is accompanied by HDL dysfunction including decreased cholesterol efflux capacity as early as in childhood and progressing in parallel to the loss of renal function.[8, 22-24] In line with this we observed that cholesterol efflux 
capacity was lower in moderate renal insufficiency patients and even more decreased in patients with severe renal insufficiency.

The association between cholesterol efflux capacity and cardiovascular mortality was only slightly weakened by inclusion of eGFR or uromodulin within the respective models indicating that there is no direct link between cholesterol efflux and kidney function. There was no interaction between cholesterol efflux on the one hand and eGFR or uromodulin on the other hand for predicting cardiovascular mortality. Consistently, the residuals of a linear regression analysis of cholesterol efflux on eGFR or uromodulin were associated with CVD mortality as strongly as crude cholesterol efflux. Furthermore, genetic variants associated with eGFR or uromodulin were associated with cholesterol efflux, but not with CVD mortality. Taken together, cholesterol efflux and kidney function are modulating cardiovascular risk by different and independent mechanisms.

A limitation of our study is that measurement of cholesterol efflux capacity does not reflect the whole reverse cholesterol transport. However, cholesterol efflux is the first major step within this pathway and the technology used has been shown to be useful in several clinical studies by us and by others. $[19,25$, 26] Additionally, our study group consisted of patients referred to coronary angiography. Therefore, our findings cannot be translated to the general population. Finally, lipoprotein parameters and cholesterol efflux capacity were measured once at baseline. We were not able to adjust for possible moderate fluctuations of these parameters during the follow-up.

Major strengths of this work are the detailed clinical and metabolic characterization of the LURIC participants and the long duration of the follow-up with a large number of fatal cardiovascular events. Another advantage was the availability of genetic data. Accordingly, we were able to confirm the relationship between cholesterol efflux and kidney function by analyzing the association between efflux capacity and SNPs which have been shown to be associated with eGFR in a meta-analysis of genomewide association studies combining data from 133,413 individuals. ${ }^{21}$

\section{Conclusions}

Our study supports the concept of dysfunctional HDL in chronic kidney disease patients. We found a strong association of cholesterol efflux capacity with kidney function as well as with cardiovascular mortality. However, detailed analyses of our data contradict the assumption that cholesterol efflux is mediating the effect of kidney function on cardiovascular outcomes. We are therefore proposing the view that cholesterol efflux and kidney function are exerting their effects on cardiovascular mortality via different and independent pathways.

\section{Declarations}

\section{Ethics approval and consent to participate}


The study was approved by the Ethics Committee at the "Aerztekammer Rheinland-Pfalz" and was performed conform to the declaration of Helsinki (837.255.97 [1394], approved January $\left.8^{\text {th }}, 1999\right)$.

\section{Consent for publication}

Not applicable.

\section{Availability of data and materials}

The datasets used and/or analysed during the current study are available from the corresponding author on reasonable request.

\section{Competing interests}

SZ is supported by the Else Kroener-Fresenius foundation. Uromodulin test kits were gratefully provided by the Institute of Experimental Immunology, Euroimmun AG Lübeck. Günther Silbernagel is member of an advisory board of AMGEN, Vienna, Austria; J.E.S. received travel support from Hofmann-Roche and Euroimmun.

\section{Funding}

This work was supported by the 7th Framework Program RiskyCAD (grant agreement number 305739) of the European Union, Brussels, Belgium; and by the Austrian Science Foundation FWF, Vienna, Austria (P27116-B23 to AR). TS is supported by the Deutsche Forschungsgemeinschaft (DFG).

\section{Authors' contributions}

AR, MEK and WM performed the statistical analysis in the LURIC cohort. AR wrote the manuscript. AR, $\mathrm{MH}, \mathrm{TS}, \mathrm{SZ}, \mathrm{TS}, \mathrm{GS}, \mathrm{HS}, \mathrm{JES}, \mathrm{MEK}$ and WM contributed to the interpretation of the results and reviewed/edited the manuscript. All authors have read and approved the manuscript as submitted.

\section{Acknowledgements}

Not applicable.

\section{References}

1. Speer T, Zewinger S, Fliser D: Uraemic dyslipidaemia revisited: role of high-density lipoprotein. Nephrol Dial Transplant 2013, 28(10):2456-2463.

2. Go AS, Chertow GM, Fan D, McCulloch CE, Hsu CY: Chronic kidney disease and the risks of death, cardiovascular events, and hospitalization. N Engl J Med 2004, 351(13):1296-1305.

3. Luscher TF, Landmesser U, von Eckardstein A, Fogelman AM: High-density lipoprotein: vascular protective effects, dysfunction, and potential as therapeutic target. Circ Res 2014, 114(1):171-182. 
4. Voight BF, Peloso GM, Orho-Melander M, Frikke-Schmidt R, Barbalic M, Jensen MK, Hindy G, Holm H, Ding EL, Johnson T et al: Plasma HDL cholesterol and risk of myocardial infarction: a mendelian randomisation study. Lancet 2012, 380(9841):572-580.

5. Pownall HJ, Gotto AM, Jr.: New Insights into the High-Density Lipoprotein Dilemma. Trends Endocrinol Metab 2016, 27(1):44-53.

6. Zewinger S, Speer T, Kleber ME, Scharnagl H, Woitas R, Lepper PM, Pfahler K, Seiler S, Heine GH, Marz W et al: HDL cholesterol is not associated with lower mortality in patients with kidney dysfunction. J Am Soc Nephrol 2014, 25(5):1073-1082.

7. Rader DJ, Alexander ET, Weibel GL, Billheimer J, Rothblat GH: The role of reverse cholesterol transport in animals and humans and relationship to atherosclerosis. J Lipid Res 2009, 50 Suppl:S189-194.

8. Shroff R, Speer T, Colin S, Charakida M, Zewinger S, Staels B, Chinetti-Gbaguidi G, Hettrich I, Rohrer L, O'Neill F et al: HDL in children with CKD promotes endothelial dysfunction and an abnormal vascular phenotype. J Am Soc Nephrol 2014, 25(11):2658-2668.

9. Speer T, Rohrer L, Blyszczuk P, Shroff R, Kuschnerus K, Krankel N, Kania G, Zewinger S, Akhmedov A, Shi $Y$ et al: Abnormal high-density lipoprotein induces endothelial dysfunction via activation of Tolllike receptor-2. Immunity 2013, 38(4):754-768.

10. Chindhy S, Joshi P, Khera A, Ayers CR, Hedayati SS, Rohatgi A: Impaired Renal Function on Cholesterol Efflux Capacity, HDL Particle Number, and Cardiovascular Events. J Am Coll Cardiol 2018, 72(6):698-700.

11. Gipson GT, Carbone S, Wang J, Dixon DL, Jovin IS, Carl DE, Gehr TW, Ghosh S: Impaired Delivery of Cholesterol Effluxed From Macrophages to Hepatocytes by Serum From CKD Patients May Underlie Increased Cardiovascular Disease Risk. Kidney Int Rep 2020, 5(2):199-210.

12. Kopecky C, Ebtehaj S, Genser B, Drechsler C, Krane V, Antlanger M, Kovarik JJ, Kaltenecker CC, Parvizi M, Wanner $C$ et al: HDL Cholesterol Efflux Does Not Predict Cardiovascular Risk in Hemodialysis Patients. J Am Soc Nephrol 2017, 28(3):769-775.

13. Untersteller K, MeissI S, Trieb M, Emrich IE, Zawada AM, Holzer M, Knuplez E, Fliser D, Heine GH, Marsche G: HDL functionality and cardiovascular outcome among nondialysis chronic kidney disease patients. J Lipid Res 2018, 59(7):1256-1265.

14. Ritsch A, Duerr A, Kahler P, Hunjadi M, Stojakovic T, Silbernagel G, Scharnagl H, Kleber ME, Marz W: Cholesterol Efflux Capacity and Cardiovascular Disease: The Ludwigshafen Risk and Cardiovascular Health (LURIC) Study. Biomedicines 2020, 8(11).

15. Winkelmann BR, Marz W, Boehm BO, Zotz R, Hager J, Hellstern P, Senges J, Group LS: Rationale and design of the LURIC study-a resource for functional genomics, pharmacogenomics and long-term prognosis of cardiovascular disease. Pharmacogenomics 2001, 2 (1 Suppl 1):S1-73.

16. American Diabetes A: Diagnosis and classification of diabetes mellitus. Diabetes Care 2006, 29 Suppl 1:S43-48. 
17. Inker LA, Schmid CH, Tighiouart H, Eckfeldt JH, Feldman HI, Greene T, Kusek JW, Manzi J, Van Lente $\mathrm{F}$, Zhang YL et al: Estimating glomerular filtration rate from serum creatinine and cystatin $\mathrm{C}$. N Engl J Med 2012, 367(1):20-29.

18. Steubl D, Block M, Herbst V, Nockher WA, Schlumberger W, Satanovskij R, Angermann S, Hasenau AL, Stecher L, Heemann U et al: Plasma Uromodulin Correlates With Kidney Function and Identifies Early Stages in Chronic Kidney Disease Patients. Medicine (Baltimore) 2016, 95(10):e3011.

19. Scharnagl H, Heuschneider C, Sailer S, Kleber ME, Marz W, Ritsch A: Decreased cholesterol efflux capacity in patients with low cholesteryl ester transfer protein plasma levels. Eur J Clin Invest 2014, 44(4):395-401.

20. Delgado GE, Kleber ME, Scharnagl H, Kramer BK, Marz W, Scherberich JE: Serum Uromodulin and Mortality Risk in Patients Undergoing Coronary Angiography. J Am Soc Nephrol 2017, 28(7):22012210.

21. Pattaro C, Teumer A, Gorski M, Chu AY, Li M, Mijatovic V, Garnaas M, Tin A, Sorice R, Li Y et al: Genetic associations at 53 loci highlight cell types and biological pathways relevant for kidney function. Nat Commun 2016, 7:10023.

22. Holzer M, Birner-Gruenberger R, Stojakovic T, El-Gamal D, Binder V, Wadsack C, Heinemann A, Marsche G: Uremia alters HDL composition and function. J Am Soc Nephrol 2011, 22(9):1631-1641.

23. Holzer M, Schilcher G, Curcic S, Trieb M, Ljubojevic S, Stojakovic T, Scharnagl H, Kopecky CM, Rosenkranz AR, Heinemann A et al: Dialysis Modalities and HDL Composition and Function. J Am Soc Nephrol 2015, 26(9):2267-2276.

24. Yamamoto S, Yancey PG, Ikizler TA, Jerome WG, Kaseda R, Cox B, Bian A, Shintani A, Fogo AB, Linton MF et al: Dysfunctional high-density lipoprotein in patients on chronic hemodialysis. J Am Coll Cardiol 2012, 60(23):2372-2379.

25. Ritsch A, Scharnagl H, Marz W: HDL cholesterol efflux capacity and cardiovascular events. N Engl J Med 2015, 372(19):1870-1871.

26. Rohatgi A, Khera A, Berry JD, Givens EG, Ayers CR, Wedin KE, Neeland IJ, Yuhanna IS, Rader DR, de Lemos JA et al: HDL cholesterol efflux capacity and incident cardiovascular events. N Engl J Med 2014, 371(25):2383-2393.

\section{Tables}

Due to technical limitations, table $1,2,3$ is only available as a download in the Supplemental Files section.

\section{Figures}




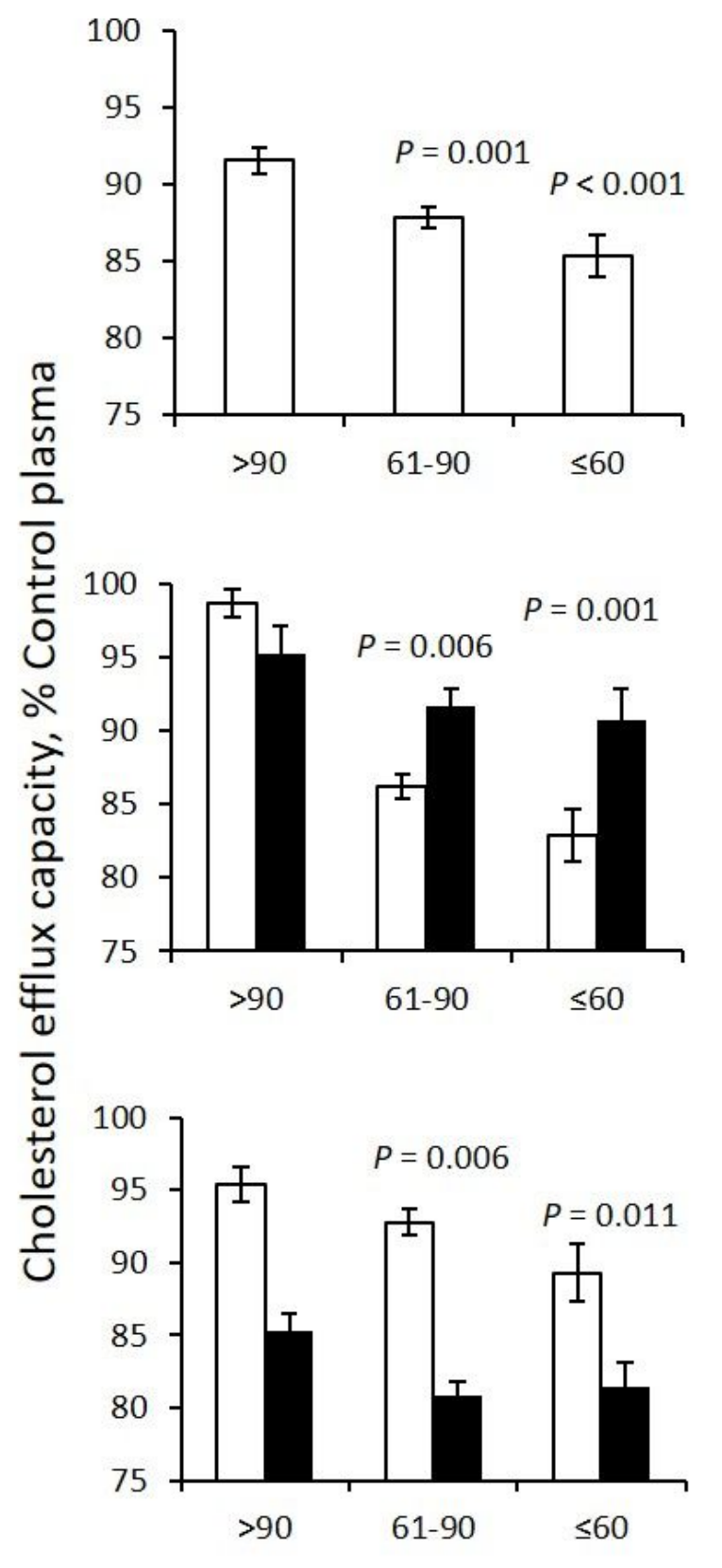

eGFR $(\mathrm{ml} / \mathrm{min} / 1.73 \mathrm{~m} 2)$

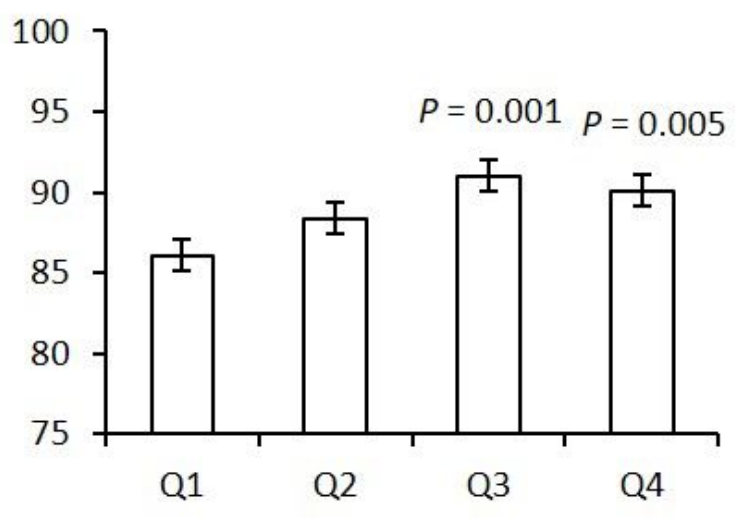

口All patients
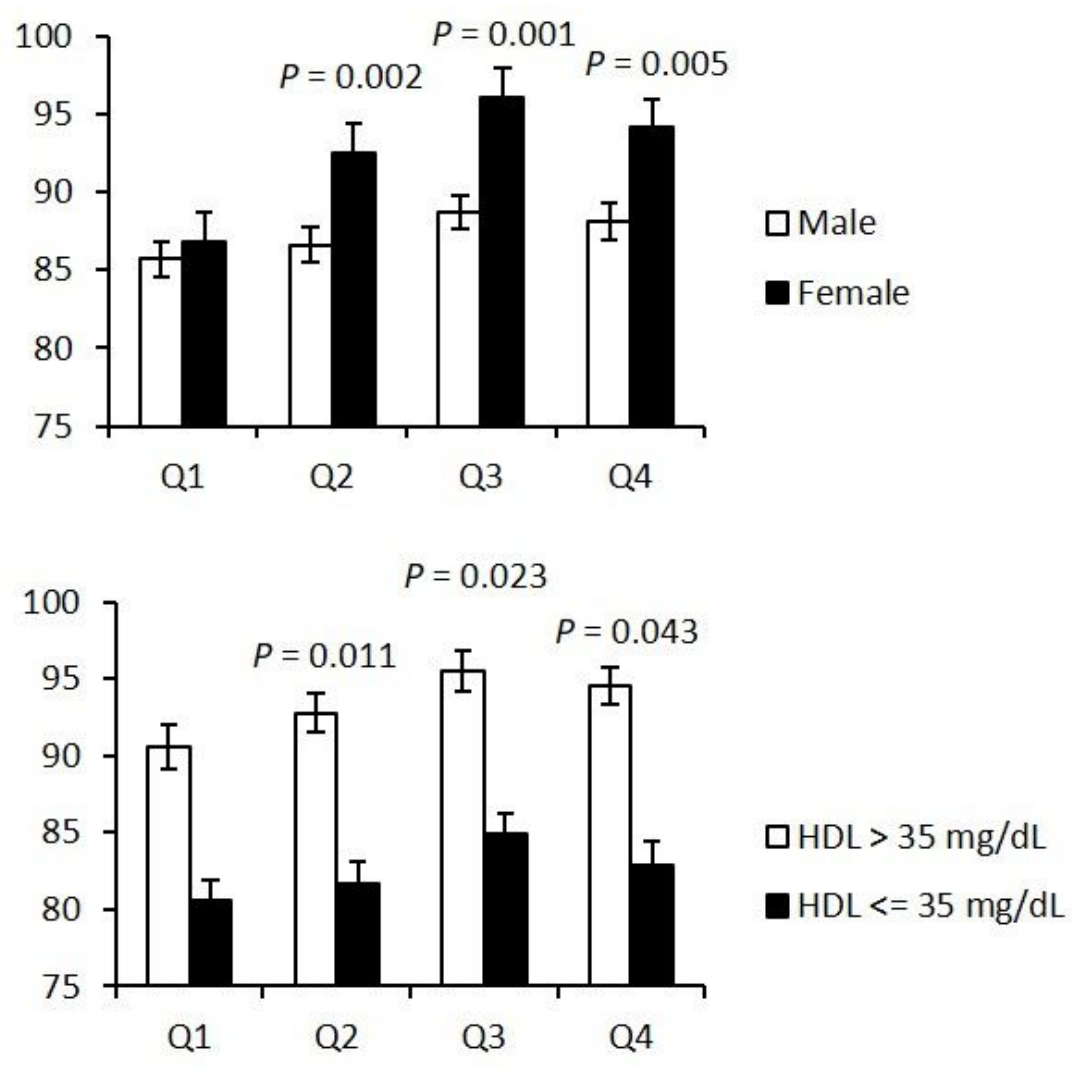

Uromodulin

Figure 1

Association of eGFR with cholesterol efflux. Multivariable adjusted estimated marginal means of cholesterol efflux capacity in sub-groups of participants of the LURIC study according to estimated glomerular filtration rate (eGFR). Subgroup analyses were performed corresponding to gender and HDL-C plasma concentration. Results are adjusted for age, gender, use of statins, CAD, BMI, diabetes mellitus, glycosylated hemoglobin, smoking, LDL/HDL cholesterol, triglycerides, ApoAl. 

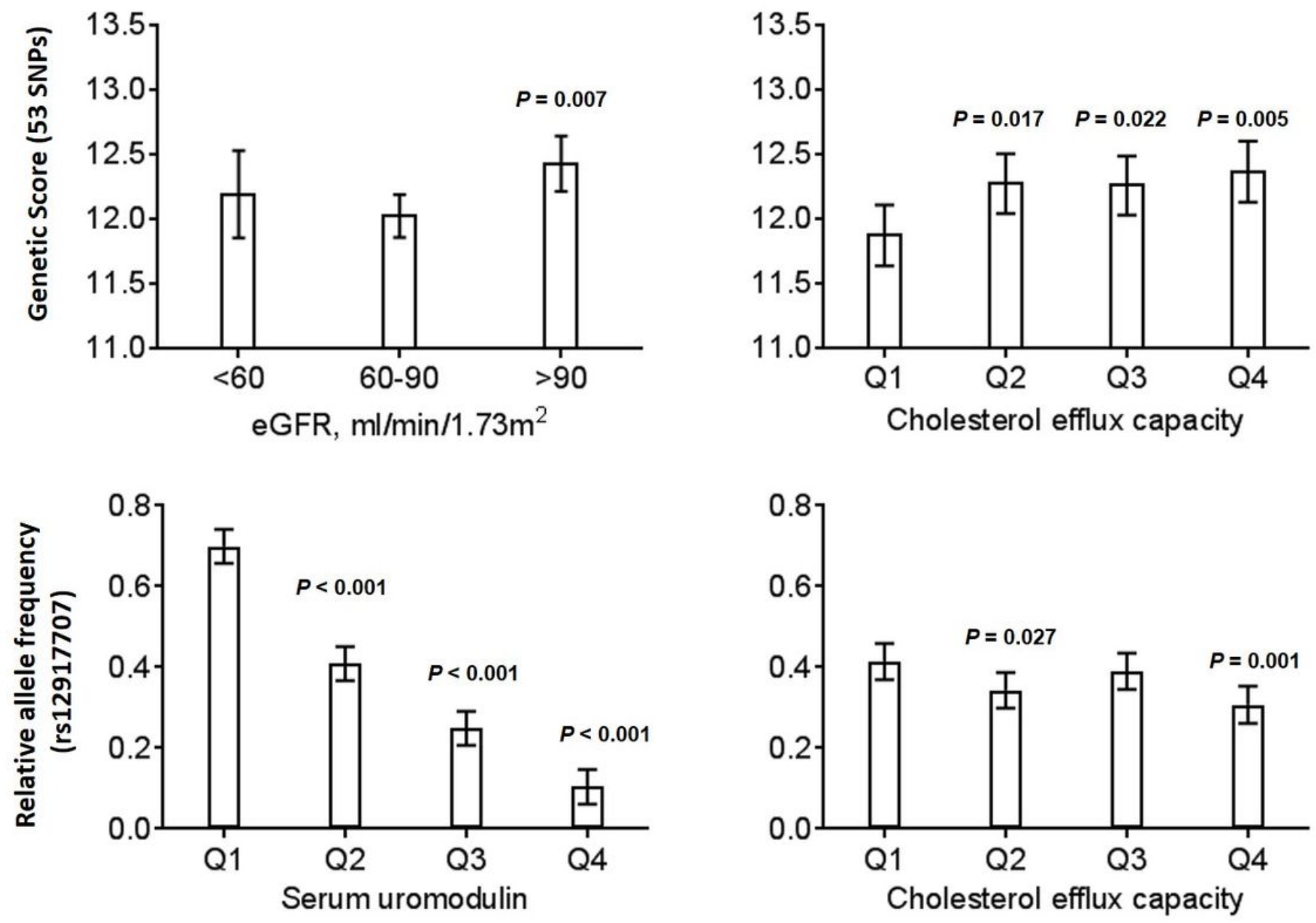

Figure 2

Association of kidney function parameters with corresponding genetic data from SNP analysis. Upper panel: Association of a genetic score of 53 independent SNPs with eGFR (left) and with cholesterol efflux capacity (right). Lower panel: Association of polymorphism rs12917707 with serum uromodulin (left) and with cholesterol efflux capacity (right). Diagrams are showing estimated marginal means and 95\% confidence intervals obtained in a general linear model, adjusted for age, gender, lipid lowering therapy, CAD-status, body mass index (BMI), diabetes mellitus, smoking history (never, former, current), hypertension, LDL-C/HDL-C ratio, apolipoprotein Al and triglycerides.

\section{Supplementary Files}

This is a list of supplementary files associated with this preprint. Click to download.

- Table1.jpg

- Table1a.jpg

- Table2.jpg

- Table2a.jpg 
- Table3.jpg

Page $13 / 13$ 